\title{
Structural Damage Identification Based on the Modal Data Change
}

\author{
Jianhua Zhao ${ }^{\mathrm{a}}$, Ling Zhang ${ }^{\mathrm{b}}$
}

MOE Key Laboratory for Strength and Vibration, Xi'an Jiaotong University, Xi'an, Shaanxi Province, China

\begin{abstract}
This paper presents a method for structural damage identification based on the modal data change before and after the occurrence of damage. In this method, it is assumed that the reduction of structural stiffness due to damage as the summation of each elemental stiffness matrix multiplied by a damage coefficient. And then, the damage coefficient (damage extent) can be solved adversely from the vibration equation. Furthermore, the modal assurance criterion (MAC) is introduced to check the correlation of mode shapes between the damaged and the undamaged structure. An advantage of employing the MAC is that it can analyze the sensitivity of mode shapes to damage. To demonstrate the capability of the proposed method, an example of a six-span planar truss beam is used to verify the present method numerically. Results indicate that the proposed method is effective in locating single or multiple damage locations and quantifying damage extent in the truss structure.
\end{abstract}

Index Terms: structural damage identification; mode shape; natural frequency; modal assurance criterion (MAC); damage coefficient

(C) 2012 Published by MECS Publisher. Selection and/or peer review under responsibility of the Research Association of Modern Education and Computer Science.

\section{Introduction}

It is truly that almost all structures are inevitably subjected to deterioration and accumulation damage during its service life due to various reasons such as environmental erosion, operating loads, fatigue, accidental bumping, etc. [1]. If the damage is not detected as early as possible, it may gradually lead to collapse or even cause catastrophe. In order to ensure the safety and reliability of the structure, many techniques for identifying damage in a structure have developed in the past decades.

Generally, structural damage is regarded as a change in the physical properties (mass, damping, and stiffness) of the structure that adversely affects its modal parameters (natural frequencies and mode shapes). In other words, the existence of structural damage may result in changes in the dynamic characteristics of a structure. Therefore, structural damage identification techniques based on modal data have drawn much more attention in recent years,

* Corresponding author.

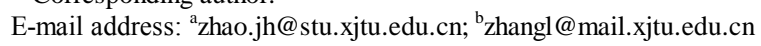


and a lot of approaches have been introduced in literature. Salawu [2] presents an overview of structural damage identification through frequency changes before and after the occurrence of damage. In practice natural frequency changes alone may not be sufficient for a unique identification of the location of structural damage. Because cracks with similar crack lengths but at two different positions may cause the same amount of frequency change. Bicanic et al. [3] proposes a novel procedure for damage identification of framed structures, where both the location and the extent of structural damage in framed structures can be correctly determined using only a limited number of measured natural frequencies. Chinchalkar [4] describes a numerical method for determining the location of a crack in a beam of varying depth when the lowest three natural frequencies of the cracked beam are known. Kim et al. [5] presents a methodology to non-destructively determine the damage location by a frequency-based method and estimate the size of damage by a mode-shape-based method in structures, respectively. The simulation result indicates that the proposed method correctly localize the damage and accurately estimate the sizes of the cracks simulated in the test beam. Ahmadia et al. [6] establishes two indicators that based on the changes in the substructure mode shapes for damage location. The change of modal characteristics can directly provide an indication of structural damage. Based on changes in frequencies and mode shapes of vibration, a new damage identification technique is proposed for predicting damage location and severity by Ren et al. [7]. Furthermore, the proposed scheme is applied to the experimental data and the consequent results are compared [8].

Although most of methods mentioned above have been much development in this field, many problems should be resolved in the practical application. For example, due to the restriction of testing equipment, a few lower-order modal parameters have been extracted. And not all modal data are sensitive to the local damage of structure. In this paper, a method considered the sensitivity of mode shapes is proposed to achieve identification of damaged location and extent. The presented approach is verified by an example of a six-span planar truss beam. Results indicate that the proposed method is effective to identify the structural damage.

\section{Theoretical Formulation}

\subsection{Basic Equations}

As is well known, the characteristic equation for an undamaged structure with no damping can be expressed as:

$$
K_{u} \phi_{u i}=\lambda_{u i} M_{u} \phi_{u i} \quad i=1, \ldots, N_{m}
$$

where $K_{u}$ and $M_{u}$ are the $n \times n$ stiffness and mass matrices, $\lambda_{u i}$ and $\phi_{u i}$ are the $i$ th Eigenvalue (the square of the natural frequency) and the corresponding eigenvector (mode shape) for the undamaged structure, $N_{m}$ is the total order of obtained mode shapes.

Similarly, without considering the influence of damping and the characteristic equation for a damaged structure is now written as follows:

$$
K_{d} \phi_{d i}=\lambda_{d i} M_{d} \phi_{d i} \quad i=1, \ldots, N_{m}
$$

where $K_{d}$ and $M_{d}$ are, respectively, the globe stiffness matrix and mass matrix of the damaged structure, $\lambda_{d i}$ and $\phi_{d i}$ are the $i$ th eigenvalue and the $i$ th eigenvector for the damaged structure.

Assuming that the mass matrix remains unchanged before and after the damage occurs, and damage can only cause changes of the stiffness matrix by an amount $\Delta K$, which can be given by 


$$
\begin{aligned}
& M_{u}=M_{d} \\
& K_{d}=K_{u}-\Delta K
\end{aligned}
$$

Substituting Equation (3) and (4) into (2) and left-Multiplying $\phi_{u i}^{T}$, yields

$$
\phi_{u i}^{T} K_{u} \phi_{d i}-\phi_{u i}^{T} \Delta K \phi_{d i}=\lambda_{d i} \phi_{u i}^{T} M_{u} \phi_{d i}
$$

Taking (1) transpose (the matrices of mass and stiffness are all considered symmetric) and right-multiplying $\phi_{d i}$, then we can get

$$
\phi_{u i}^{T} K_{u} \phi_{d i}=\lambda_{u i} \phi_{u i}^{T} M_{u} \phi_{d i}
$$

Substituting (6) into (5) and rearranging it, we obtain

$$
\Delta \lambda_{i}=\lambda_{u i}-\lambda_{d i}=\frac{\phi_{u i}^{T} \Delta K \phi_{d i}}{\phi_{u i}^{T} M_{u} \phi_{d i}}
$$

where $\Delta \lambda_{i}$ denotes the change of natural frequency when the damage occurs.

So far, there is no a general model of damage. It is further assumed that any occurrence of structural damage causes a local change in stiffness and the reduction of structural stiffness due to damage as the summation of each elemental stiffness matrix multiplied by a damage coefficient, the perturbation $\Delta K$, can be written as [9]

$$
\Delta K=\sum_{j=1}^{N_{e}} \alpha_{j} K_{j} \quad\left(0 \leq \alpha_{j} \leq 1\right)
$$

where $K_{j}$ and $\alpha_{j}$ are the jet elemental stiffness matrix and its damage extent, respectively; $N_{e}$ is the total number of elements.

Substituting (8) into (7) and it can be rewritten in simplified form

$$
\Delta \lambda=D \alpha
$$

in which

$$
\Delta \lambda=\left[\Delta \lambda_{1}, \Delta \lambda_{2}, \cdots, \Delta \lambda_{m}\right]^{T}
$$

and 


$$
\alpha=\left[\alpha_{1}, \alpha_{2}, \cdots, \alpha_{N_{e}}\right]^{T}
$$

and

$$
D=\left[\begin{array}{cccc}
\frac{\phi_{u 1}^{T} K_{1} \phi_{d 1}}{\phi_{u 1}^{T} M_{u} \phi_{d 1}} & \frac{\phi_{u 1}^{T} K_{2} \phi_{d 1}}{\phi_{u 1}^{T} M_{u} \phi_{d 1}} & \cdots & \frac{\phi_{u 1}^{T} K_{N_{e}} \phi_{d 1}}{\phi_{u 1}^{T} M_{u} \phi_{d 1}} \\
\frac{\phi_{u 2}^{T} K_{1} \phi_{d 2}}{\phi_{u 2}^{T} M_{u} \phi_{d 2}} & \frac{\phi_{u 2}^{T} K_{2} \phi_{d 2}}{\phi_{u 2}^{T} M_{u} \phi_{d 2}} & \cdots & \frac{\phi_{u 2}^{T} K_{N_{e}} \phi_{d 2}}{\phi_{u 2}^{T} M_{u} \phi_{d 2}} \\
\vdots & \vdots & \ddots & \vdots \\
\frac{\phi_{u m}^{T} K_{1} \phi_{d m}}{\phi_{u m}^{T} M_{u} \phi_{d m}} & \frac{\phi_{u m}^{T} K_{2} \phi_{d m}}{\phi_{u m}^{T} M_{u} \phi_{d m}} & \cdots & \frac{\phi_{u m}^{T} K_{N_{e}} \phi_{d m}}{\phi_{u m}^{T} M_{u} \phi_{d m}}
\end{array}\right]
$$

where, subscript " $m$ " indicates the number of measured mode shapes. (9) characterizes the relationship between the damage extent and the modal characteristic of the structure and the change in the structural parameter at the elemental level.

From (9), the damage extent vector can be calculated as

$$
\alpha=D^{+} \Delta \lambda
$$

where, superscript “+” denotes the Moore-Penrose generalized inverse which can be solved by singular value decomposition.

\subsection{Modal Assurance Criterion (MAC)}

Among the modal parameters of a structure system, the mode shape is obviously the only location-related parameter [10]. Mode shape is more sensitive to local damage than modal frequencies, especially the higher modes. In other words, it may provide more information of structural damage. The index MAC is usually employed to indicate the correlation between two sets of mode shapes, for example, those from damaged and undamaged models. The MAC is defined as [11]

$$
\operatorname{MAC}\left(\phi_{u i}, \phi_{d i}\right)=\frac{\left|\phi_{u i}^{T} \phi_{d i}\right|^{2}}{\left(\phi_{u i}^{T} \phi_{u i}\right)\left(\phi_{d i}^{T} \phi_{d i}\right)}
$$

where $\phi_{u i}$ is the $i$ th mode shape of the undamaged structure and $\phi_{d i}$ is the corresponding mode shape of the damaged structure. The value of MAC is between 0 and 1 . When the two sets of mode shapes fit each other well, the value is close to 1 . That is to say, there is no an obvious change in mode shape before and after the occurrence of damage. Meanwhile, a value of 0 means no correlation. It can be interpreted that the mode shape is sensitive to the damage. For the purpose of structural damage identification, the modal parameters should be sensitive to even slight local structural damage. The MAC as an index can check the correlation between the two sets of mode shapes before and after the damage occurs. So the MAC is used to analyse the sensitivity of mode shapes. 


\section{Numerical Example}

A six-span planar truss (shown in Fig. 1) is adopted to verify the performance of the proposed method. The material properties and geometric parameters of the structures are as follows: elastic modulus $E=200 \mathrm{GPa}$, Poisson's ratio $v=0.3$, mass density $\rho=7.8 \times 103 \mathrm{Kg} / \mathrm{m}^{3}$, the length of each element $l=0.5 \mathrm{~m}$, cross-sectional area $A=0.0025 \mathrm{~m}^{2}$. The finite element model of the truss consists of 14 nodes and 31 elements. The total number of degrees of freedom is 25 . Assuming the finite element model can accurately simulate the actual dynamic characteristics of the structure, and the measured data are also generated from the finite element model of the structure. Three damage cases are analysed as shown in table I. Damages are simulated by a reduction in the stiffness of the elements. The first eight natural frequencies for the intact and damaged structures are listed in table II.

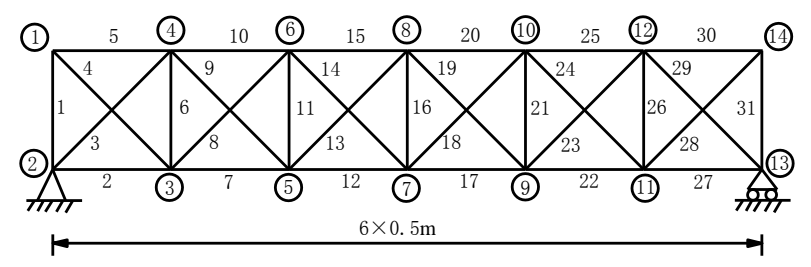

Fig. 1. A planar truss model

Table 1. damage case for the planar truss

\begin{tabular}{ccc}
\hline Damage case & Damage element & Damage extent \\
\hline A & 10 & $30 \%$ reduction in E \\
B & 9,17 & $20 \%$ reduction in E \\
C & $6,17,18$ & $30 \%, 30 \%, 20 \%$ reduction in E \\
\hline
\end{tabular}

Table 2. Natural frequencies of the intact and damaged truss beam

\begin{tabular}{ccccc}
\hline \multirow{2}{*}{ Mode } & \multicolumn{4}{c}{ Frequencies/Hz } \\
\cline { 2 - 5 } & Intact & Case A & Case B & Case C \\
\hline 1 & 111.56 & 110.27 & 108.27 & 108.41 \\
2 & 233.02 & 232.04 & 232.81 & 231.81 \\
3 & 409.75 & 398.60 & 405.56 & 403.97 \\
4 & 684.03 & 681.33 & 662.01 & 662.74 \\
5 & 766.11 & 763.22 & 763.84 & 763.49 \\
6 & 1100.4 & 1091.7 & 1077.1 & 1091.1 \\
7 & 1141.8 & 1119.2 & 1121.8 & 1133.6 \\
8 & 1358.2 & 1355.1 & 1353.7 & 1346.1 \\
\hline
\end{tabular}

The correlation between mode shapes for the intact structure and the damaged structure is checked by the MAC values, results shown in Fig. 2. It is noteworthy to mention that the MAC values have a large change between different orders. In other words, not all orders of mode shapes are sensitive to the local structural damage. Therefore, it may choose some best mode shapes to identify the damage. For case A, it is obviously that the MAC values of the 4th, 6th and 7th-order modes are smaller than any other ones. So they are used to calculate damage 
coefficient. Similarly, for case B and case C, we also choose the smaller ones to locate the damage position and estimate the damage severity.

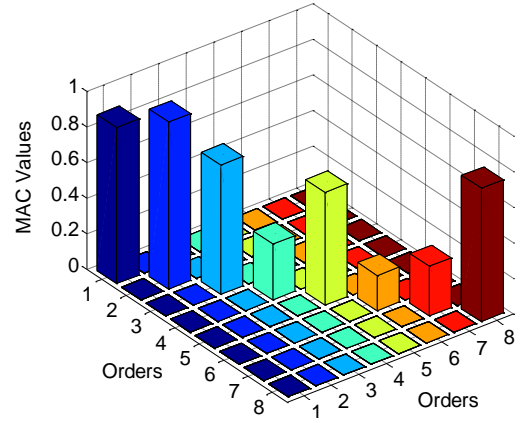

(a) Case $\mathrm{A}$

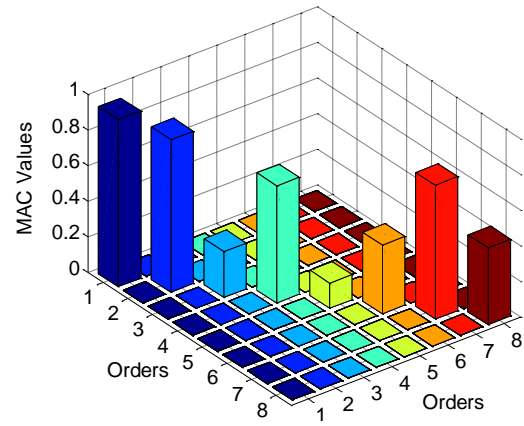

(b) Case B

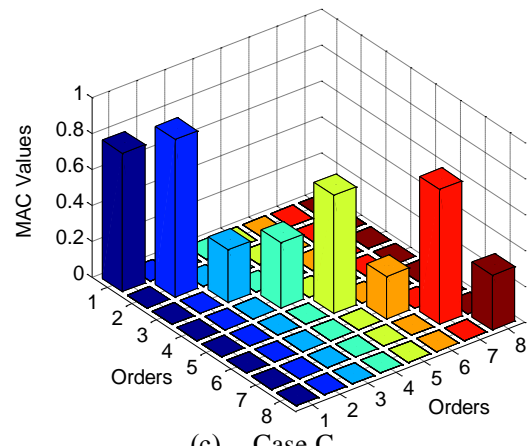

Fig. 2. MAC values for damaged and undamaged structures

The identified results of three damage cases are shown in Fig. 3. For case A, it assumes a single damage occurs in the 10th element with a stiffness lost of $30 \%$. The 4 th, 6 th and 7 th-order modes are used to calculate the damage extent. From Fig. 3(a), it is easily to determine the damage location. Simultaneously, the damage coefficient can be obtained as $\alpha_{10}=0.2874$ by using (10). The relative error between the identified result and the actual value is only 4.2\%. Case B has double damages that occur in elements 9 and 17 with a loss of stiffness of $20 \%$ in both of them, and the damaged members can also be found easily as it is shown in Fig. 3(b). Using the 3rd, 5th, 6th and 8th-order modes to solve (10), it can obtain the values of damage extent in the elements 9 and 17 are $\alpha_{9}=0.1864$ 
and $\alpha_{17}=0.1904$, respectively. Correspondingly, the relative errors between the identified result and the actual value are $6.8 \%$ and $4.8 \%$. Case $\mathrm{C}$ is used to demonstrate the ability of the proposed method to identify the multiple damages. Results computed using data from the 3rd, 4th, 6th and 8th-order modes are shown in Fig. 3(c). The identified solutions are $\alpha_{6}=0.2796, \alpha_{17}=0.2835$ and $\alpha_{18}=0.1861$. Moreover, compared to the actual damage extent, the relative errors are $6.8 \%, 5.5 \%$ and $6.95 \%$, respectively. From the above results, it can be seen that the present damage identification method is effective to locate single or multiple damage locations and quantify damage extent in the truss structure. In addition, it should be noted that the relative error increases as the multiple damages occur in a structure.

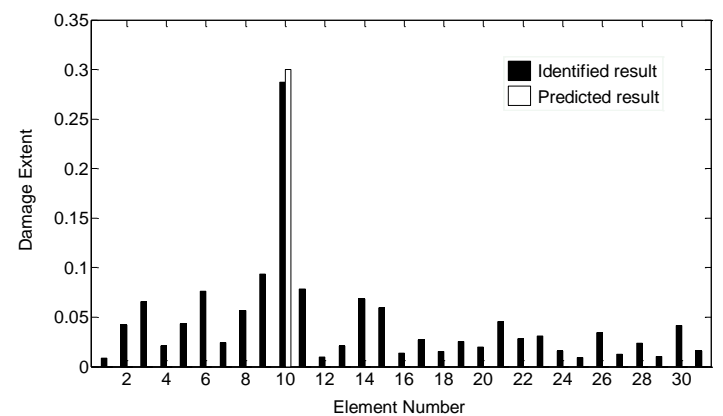

(a) Case $\mathrm{A}$

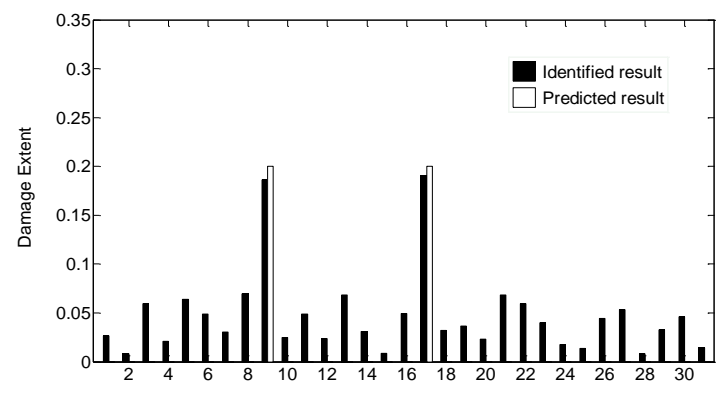

(b) Case B

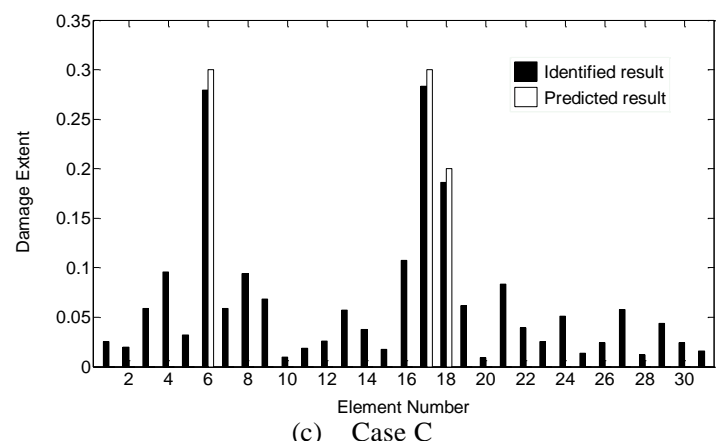

Fig. 3. Damage identification results 


\section{Conclusions}

In this paper, a damage identification approach based on the modal data change for truss beam has been developed. It relies on the fact that existence of structural damage may lead to changes in the modal parameters (natural frequencies and mode shapes). In this method, damage to a structure can be considered as the summation of each elemental stiffness matrix multiplied by a damage coefficient. The damage coefficient as a damage index can represent the damage location and severity. And then, the perturbation of stiffness matrix is substituted into vibration equation to solve damage coefficient adversely. In order to check the correlation of mode shapes between the damaged and the undamaged structure, the modal assurance criterion (MAC) is introduced. Furthermore, the MAC is used to analyse the sensitivity of mode shapes once the damage occurs. Results of numerical examples show that the proposed method is capable of identifying the location and extent of damage in the truss structure. However, environmental noise and measurement errors are inevitable in practice. So further studies will be focus on validating the methodology by laboratory tests for large-scale and complex structures.

\section{References}

[1] Y. Xia and H. Hao, "Statistical damage identification of structures with frequency changes," Journal of Sound and Vibration, vol. 263, pp. 853-870, 2003.

[2] O. S. Salawu, "Detection of structural damage through changes in frequency: a review," Engineering Structures, vol. 19, no. 9, pp.718-723, Sep 1997.

[3] N. Bicanic and H. P. Chen, "Damage identification in framed structures using natural frequencies," International Journal for Numerical methods in Engineering, vol. 40, pp. 4451-4468, Feb 1997.

[4] S. Chinchalkar, "Determination of crack location in beams using natural frequencies," Journal of Sound and Vibration, vol. 247, no. 3, pp. 417-429, March 2001.

[5] J. T. Kim, Y. S. Ryu, H. M. Cho, and N. Stubbs, "Damage identification in beam-type structures: frequency-based method vs mode-shape-based method," Engineering Structures, vol. 25, pp. 57-67, 2003.

[6] H. Ahmadian, J. E. Mottershead, and M. I. Friswell, "Damage location indicators from substructure mode shapes," Inverse Problems in Science and Engineering, vol. 8, no. 4, pp. 309-323, Apr 2000.

[7] W. X. Ren and G. D. Roeck, "Structural damage identification using modal data. I: simulation verification," Journal of Structural Engineering, ASCE, vol. 128, no. 1, pp. 87-95, Jan 2002.

[8] W. X. Ren and G. D. Roeck, "Structural damage identification using modal data. II: test verification," Journal of Structural Engineering, ASCE, vol. 128, no. 1, pp. 96-104, Jan 2002.

[9] Z. Y. Shi, S. S. Law, and L. M. Zhang, "Structural damage detection from modal strain energy change," Journal of Engineering Mechanics, ASCE, Vol. 126, no. 12, pp.1216-1223, December 2000.

[10] J. F. Wang, C. C. Lin, and S. M. Yen, "A story damage index of seismically-excited buildings based on modal frequency and mode shape," Engineering Structures, Vol. 29, pp.2143-2157, 2007.

[11] R. J. Allemang and D. L. Brown, "A correlation coefficient for modal vector analysis," Proceedings of the first international modal analysis conference, Orlando, FL, pp. 110-116, 1982. 